\title{
Surgical Manipulation of the Occlusal Plane in Class III Deformities: 5 Features to Help Planning
}

\author{
Eduardo Parente1, Guilherme Lacerda ${ }^{2}$, Marcelo Galindo Silvares ${ }^{{ }^{*}}$ \\ ${ }^{1}$ Department of Oral and Maxillofacial Surgery, State University of Rio de Janeiro, Rio de Janeiro, Brazil \\ ${ }^{2}$ Department of Oral and Maxillofacial Surgery, Pontifical Catholic University of Minas Gerais PUC, Belo \\ Horizonte, Brazil \\ Email: ${ }^{*}$ marcelogalindos@gmail.com
}

Received 12 March 2014; revised 17 April 2014; accepted 25 April 2014

Copyright (C) 2014 by authors and Scientific Research Publishing Inc.

This work is licensed under the Creative Commons Attribution International License (CC BY). http://creativecommons.org/licenses/by/4.0/

c) (i) Open Access

\begin{abstract}
Class III malocclusion is one of the most common deformities that requires correction through orthodontic-surgical treatment. These patients present different facial patterns depending on the occlusal plane angulation. These patients may benefit from surgical manipulation of the occlusal plane, because only bite correction does not always lead to the improvement of the facial deformity. The purpose of this paper is to show the surgical manipulation of the occlusal plane in Class III patients based on five features: 1) facial type, 2) upper incisor display at rest, 3) dental exposure during smile, 4) soft tissue support, and 5) chin projection. The surgical manipulation of the occlusal plane can improve the esthetic outcome in the treatment of class III deformities, and careful evaluation of the five features described could be a helpful planning method.
\end{abstract}

\section{Keywords}

Orthognathic Surgery, Occlusal Plane Alteration, Class III Malocclusion

\section{Introduction}

One of the most common indications for surgical-orthodontic treatment is Class III deformity. However, these patients present with different facial types, and the occlusal plane angle manipulation has been one of the most useful pieces of data to achieve better outcomes [1].

The normal occlusal plane for adults is $8^{\circ}$ on average and a deviation reflects in the facial pattern, regardless

"Corresponding author. 
of the malocclusion type [2]-[5]. Commonly, Class III high occlusal plane facial type (HOP) patients show an increased occlusal plane, long face and decreased projection of the chin relative to mandibular incisor (Figure 1), while Class III low occlusal plane facial type (LOP) patients show opposite clinical findings (Figure 2) [2]-[7]. These patients may benefit from surgical manipulation of the occlusal plane, because only bite correction does not always lead to the improvement of the facial deformity [1]-[3] [8].

Malocclusion classification for surgical and orthodontic treatment was enhanced with Ackerman and Proffit's suggestion of completing dental and skeletal evaluation in the 3 planes of space and involving soft and hard tissue relationships [9] [10].

A harmonious facial and dental appearance is the goal of orthodontists and surgeons when treating a patient. The purpose of this paper is to show the surgical manipulation of the occlusal plane in Class III patients based on five features: 1) facial type, 2) upper incisor display in repose, 3) dental exposure during smile, 4) labial, nasal and paranasal support, and 5) chin projection relative to mandibular incisors.

\section{Occlusal Plane Manipulation According to the Five Features}

The five features are observed with the patient in natural head position and the data are recorded. The facial type is the main determinant in the type of rotation. Patients with LOP usually have better results with clockwise ro-

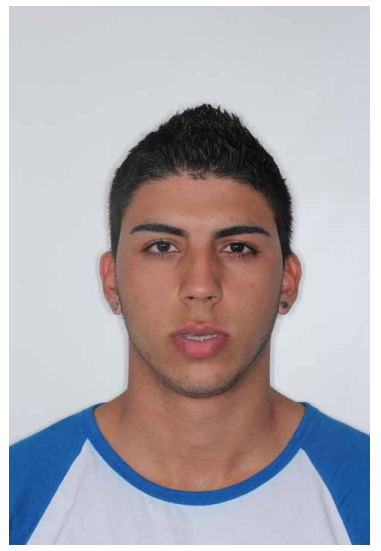

(a)

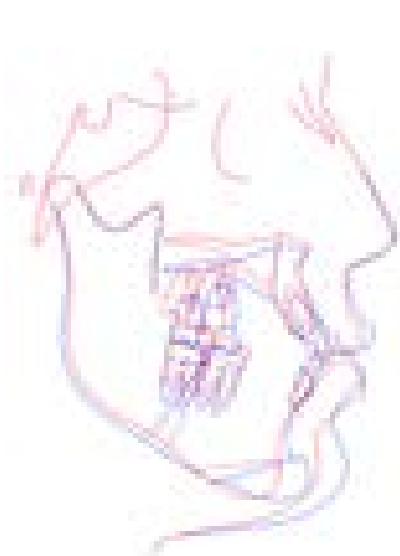

(e)

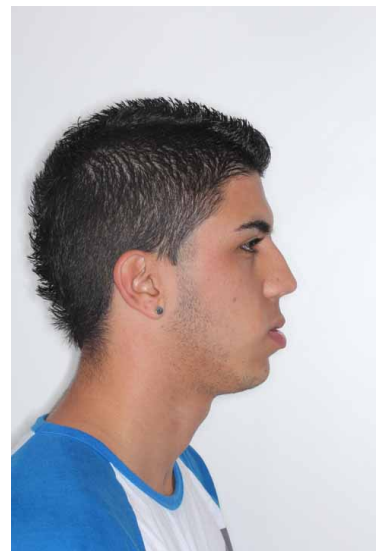

(b)

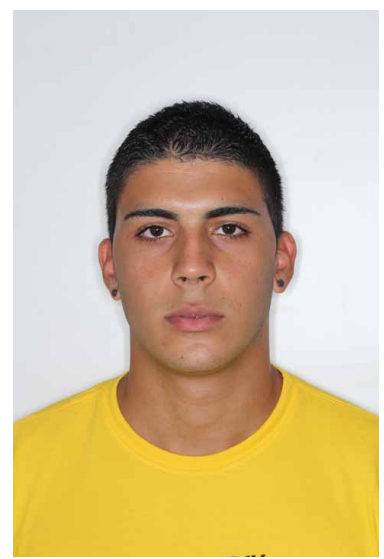

(f)

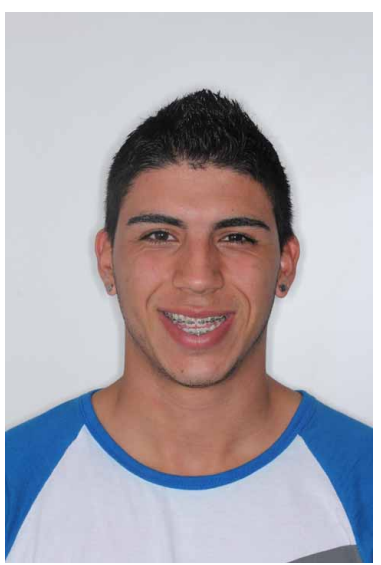

(c)

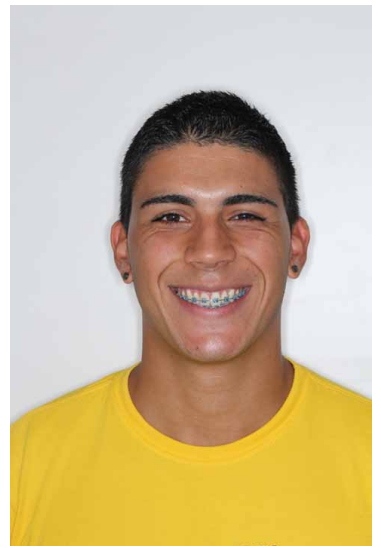

(g)

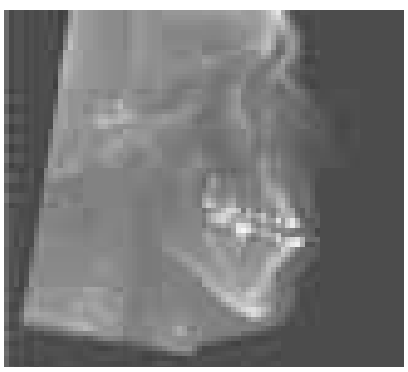

(d)

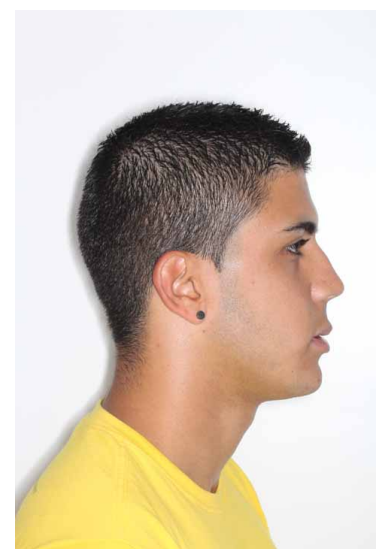

(h)

Figure 1. 18-year-old patient that presented a HOP with class III malocclusion, underwent a counterclockwise rotation. (a) Preoperative frontal view showing a long face appearance, without vertical maxillary excess; (b) Preoperative profile view showing significant decrease of chin projection relative to the lower lip; (c) Preoperative smile view evidencing reduced gingival exposure at the posterior area of the maxilla, allowing the inferior reposition of this area; (d) Preoperative lateral cephalometric showing an increased occlusal plane and decreased projection of the chin relative to mandibular incisor; (e) Initial and predict tracing superimposition; (f)-(h) Five months follow-up showing good esthetic results after maxillomandibular counterclockwise rotation associated with vertical reduction and advancement genioplasty. 


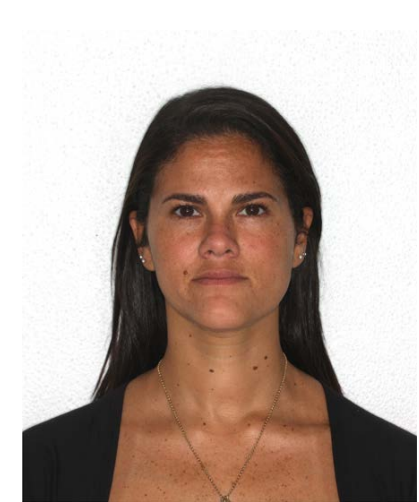

(a)

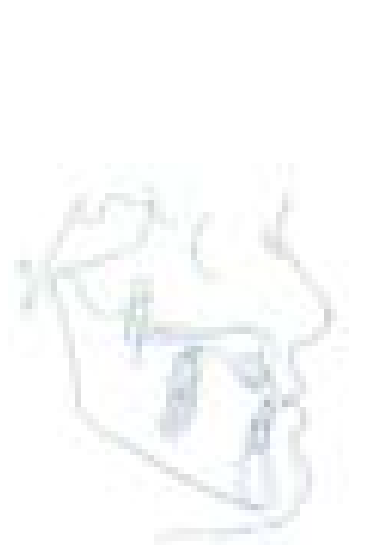

(e)

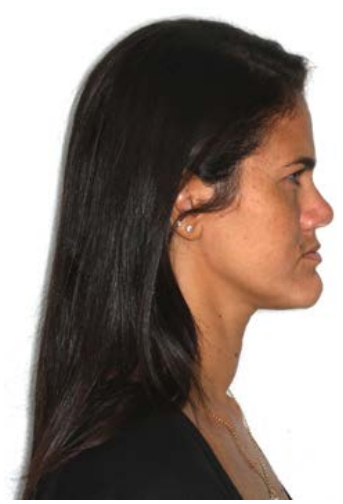

(b)

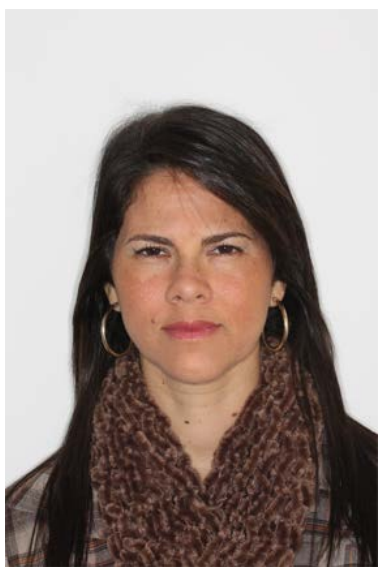

(f)

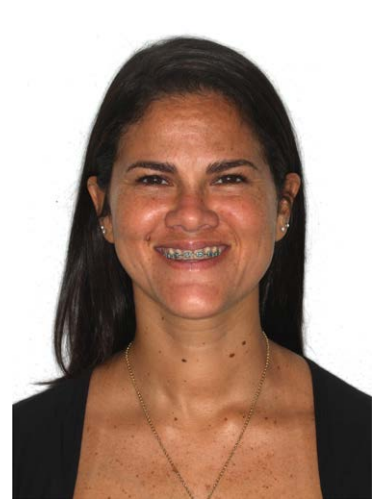

(c)

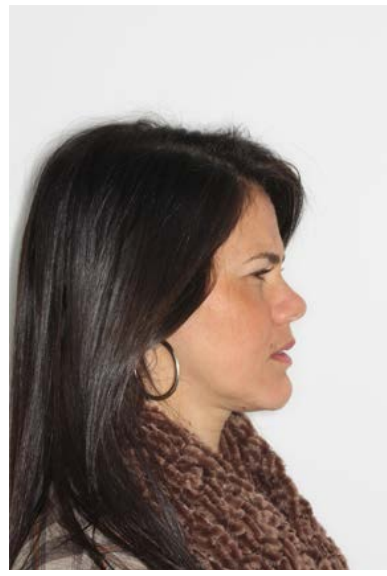

(g)

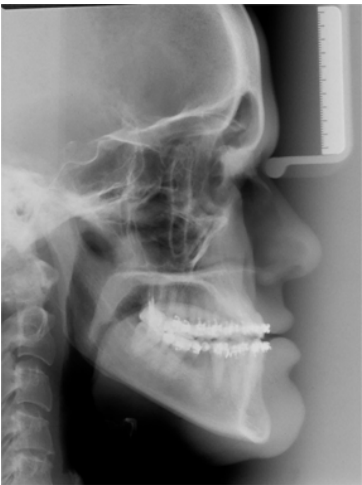

(d)

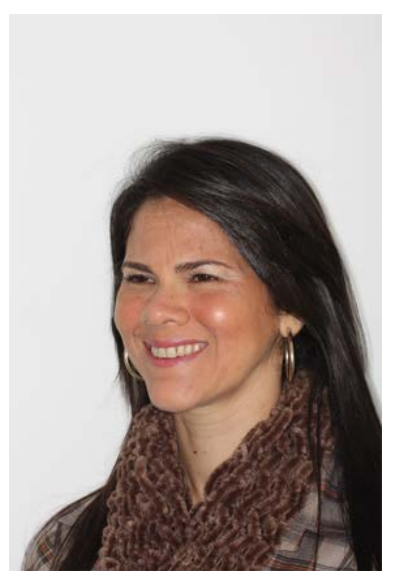

(h)

Figure 2. 31-year-old patient that presented a LOP facial type with a class III malocclusion, underwent to a clockwise rotation of the maxillomandibular complex. (a) Preoperative frontal view evidencing a square-jaw appearance and facial aging; (b) Preoperative profile view showing the lack of paranasal support and excessively projection of the chin; (c) Preoperative smile view showing greater gingival exposure at the posterior area of the maxilla; (d) Preoperative lateral cephalometric showing a decreased occlusal plane and increased projection of the chin relative to mandibular incisor; (e) Initial and predict tracing superimposed; (f)-(h) Sixteen months follow-up.

tation because the chin rotates posteriorly, the mandibular plane angle increases and the mandibular contour is softened. On the other hand, HOP patients benefit of a counter-clockwise rotation because the chin rotates anteriorly, the mandibular plane angle decreases, the prominence of the mandibular angles increases and mandibular contour is improved.

The anteroposterior tilt of the occlusal plane (pitch), determines tooth and gingival display at rest and during smile. This is the main characteristic to evaluate the degree of maxillary vertical reposition needed. This evaluation is performed with profile, frontal, and $45^{\circ}$ views and should include the clinical crown height of the teeth, the level of the gingival margins and the smile arc to optimize both dental and facial appearance. Upper incisor display should be measured in repose (if the display is negative, lateral cephalometric radiograph with open mouth can be used) and smiling to guide anterior vertical repositioning; molars and premolars display should be measured during smile to guide posterior vertical repositioning. The authors usually formulate the surgical plan to obtain approximately $3 \mathrm{~mm}$ of display of the central incisors at rest, with the elevation of the lip stopping at the gingival margins of the upper teeth during smile. However, some amount of gingival display is certainly acceptable and the final plan will be a balance between these clinical findings.

Lack of soft tissue support in the mid face is observed from two different aspects: labial and nasal/paranasal support. Owing to the fact that lip support is improved with dental advancement and nasal/paranasal support with bony advancement, each type of rotation will have more of an effect on one or the other. The amount of occlusal plane manipulation and maxillary advancement should be enough to reach the ideal soft tissue support. 
Usually, the authors use cephalometric values as the maxillary depth and nasolabial angle to confirm the upper jaw desired position. However, the surgical reposition is mainly based on the clinical examination.

Finally, the chin projection relative to the mandibular incisor projection helps to regulate the amount of the occlusal plane manipulation: the greater the discrepancy, the greater the rotation needed. In cases where the lower incisors are too anterior relative to the chin position, a counter-clockwise rotation of the occlusal plane would be indicated. The opposite would be true in cases of too much chin projection. To obtain better cosmetic outcomes it is commonly helpful to plan the occlusal plane rotation with a horizontal osteotomy of the symphysis to avoid chin setback and also to limit the genioplasty advancement to a few millimeters.

The surgical manipulation of the occlusal plane is planned on these findings. As a routine, the other elements of facial analysis are used to refine the surgical planning and then a prediction tracing is completed and model surgery performed according to the desired treatment planning. Since the beginning of 2013 we have treated 86 patients with that technique and so far, we have not had any class III malocclusion patient where it cannot be applied.

\section{Discussion}

The surgical correction of Class III malocclusion by surgical manipulation of the occlusal plane is an interesting technique. However, the surgeon should remember that the occlusal plane angulation can show a significant variation within the population. Consequently, the so-called normal "occlusal plane angle" exists within a wide range and there is no clear functional advantage in obtaining a specific "degree" of angulation [2] [3]. The treatment plan in orthognathic surgery is required to be based on clinical features and not on numerical values. Therefore, we use a balance between the 5 features presented to achieve good cosmetic outcomes.

As the planning is mainly based on clinical evaluation, one limitation is that there is no standardization with this technique. The changes will be based on the surgeon's own clinical perceptions and should be individualized for each patient.

Two jaw surgeries without occlusal plane manipulation for Class III patients are commonly insufficient to achieve a good chin position [2] [3] [5] [7]. The clockwise and counterclockwise rotation of the maxillomandibular complex will move the chin to a better position in the low and high occlusal plane facial types, respectively [2] [3] [5] [6]. These approaches can reduce or avoid the need of genioplasty, making the final result more natural. However, care should be taken to avoid over correction of the occlusal plane angulation to achieve good chin position. This could result in undesirable effects on nasal and paranasal areas, as well as inadequate dental and gingival display during repose, speech, and smile.

In addition to the esthetic benefit, maxillomandibular counter-clockwise rotation commonly increases the pharyngeal airway dimension. However, a recent study suggests that this rotation could reduce the pharyngeal airway space when mandibular setback is performed [11]. Since this rotation moves the mandibular incisors in a backward direction and the chin in a forward direction, it usually reduces or avoids a true mandibular setback and could preserve or enlarge the pharyngeal airway dimension.

Pitch manipulation was the primary focus of this paper. Its relationship to the smile arc and esthetics plays an important role in the final treatment result [10]; however, most surgical patients present with various degrees of roll and yaw alterations that should be taken into account for the treatment plan.

Another important concern is long term stability. Studies show that selective occlusal plane alteration in clockwise and counter-clockwise direction during two jaw surgery on patients with healthy temporomandibular joints and using rigid fixation appears to be stable [2] [3] [12] [13].

\section{Conclusions}

The surgical manipulation of the occlusal plane can improve the esthetic outcome in the treatment of class III deformities, and careful evaluation of the five features described could be a helpful planning method.

Each of the features has its own balance that could be easily reachable; however, finding the perfect match for all 5 of them together is a very complicated task.

\section{References}

[1] Johnston, C., Burden, D., Kennedy, D., Harradine, N. and Stevenson, M. (2006) Class III Surgical-Orthodontic Treatment: A Cephalometric Study. American Journal of Orthodontics and Dentofacial Orthopedics, 130, 300-309.

http://dx.doi.org/10.1016/j.ajodo.2005.01.023 
[2] Wolford, L.M., Chemello, P.D. and Hilliard, F. (1994) Occlusal Plane Alteration in Orthognathic Surgery-Part I: Effects on Function and Esthetics. American Journal of Orthodontics and Dentofacial Orthopedics, 106, 304-316. http://dx.doi.org/10.1016/S0889-5406(94)70051-6

[3] Posnick, J.C., Fantuzzo, J.J. and Orchin, J.D. (2006) Deliberate Operative Rotation of the Maxillo-Mandibular Complex to Alter the A-Point to B-Point Relationship for Enhanced Facial Esthetics. Journal of Oral and Maxillofacial Surgery, 64, 1687-1695. http://dx.doi.org/10.1016/j.joms.2005.11.118

[4] Arnett, G.W., Jelic, J.S., Kim, J., Cummings, D.R., Beress, A., Worley Jr., C.M., et al. (1999) Soft Tissue Cephalometric Analysis: Diagnosis and Treatment Planning of Dentofacial Deformity. American Journal of Orthodontics and Dentofacial Orthopedics, 116, 239-253. http://dx.doi.org/10.1016/S0889-5406(99)70234-9

[5] Reyneke, J.P. (1999) Surgical Cephalometric Prediction Tracing for the Alteration of the Occlusal Plane by Means of Rotation of the Maxillomandibular Complex. The International Journal of Adult Orthodontics \& Orthognathic Surgery, 14, 55-64.

[6] Reyneke, J.P. and Evans, W.G. (1990) Surgical Manipulation of the Occlusal Plane. The International Journal of Adult Orthodontics \& Orthognathic Surgery, 5, 99-110.

[7] Reyneke, J.P. (1998) Surgical Manipulation of the Occlusal Plane: New Concepts in Geometry. The International Journal of Adult Orthodontics \& Orthognathic Surgery, 13, 307-316.

[8] Arnett, G.W. and Bergman, R.T. (1993) Facial Keys to Orthodontic Diagnosis and Treatment Planning. Part I. American Journal of Orthodontics and Dentofacial Orthopedics, 103, 299-312. http://dx.doi.org/10.1016/0889-5406(93)70010-L

[9] Ackerman, J.L. and Proffit, W.R. (1969) The Characteristics of Malocclusion: A Modern Approach to Classification and Diagnosis. American Journal of Orthodontics, 56, 443-454. http://dx.doi.org/10.1016/0002-9416(69)90206-1

[10] Ackerman, J.L., Proffit, W.R., Sarver, D.M., Ackerman, M.B. and Kean, M.R. (2007) Pitch, Roll, and Yaw: Describing the Spatial Orientation of Dentofacial Traits. American Journal of Orthodontics and Dentofacial Orthopedics, 131, 305-310. http://dx.doi.org/10.1016/j.ajodo.2006.05.032

[11] Mehra, P., Downie, M., Pita, M.C. and Wolford, L.M. (2001) Pharyngeal Airway Space Changes after Counterclockwise Rotation of the Maxillomandibular Complex. American Journal of Orthodontics and Dentofacial Orthopedics, 120, 154-159. http://dx.doi.org/10.1067/mod.2001.114647

[12] Chemello, P.D., Wolford, L.M. and Buschang, P.H. (1994) Occlusal Plane Alteration in Orthognathic Surgery-Part II: Long-Term Stability of Results. American Journal of Orthodontics and Dentofacial Orthopedics, 106, 434-440. http://dx.doi.org/10.1016/S0889-5406(94)70066-4

[13] Reyneke, J.P., Bryant, R.S., Suuronen, R. and Becker, P.J. (2007) Postoperative Skeletal Stability Following Clockwise and Counter-Clockwise Rotation of the Maxillomandibular Complex Compared to Conventional Orthognathic Treatment. British Journal of Oral and Maxillofacial Surgery, 45, 56-64. http://dx.doi.org/10.1016/j.bjoms.2005.12.015 